\title{
Sensor Placement for Fault Diagnosis Performance Maximization in Distribution Networks
}

\author{
Ramon Sarrate, Fatiha Nejjari and Albert Rosich
}

\begin{abstract}
The success of any diagnosis strategy critically depends on the sensors measuring process variables. This paper presents a strategy based on diagnosability maximization for optimally locating sensors in distribution networks. The goal is to characterize and determine the set of sensors that guarantees a maximum degree of diagnosability taking into account a given sensor configuration cardinality constraint. The strategy is based on the structural model of the system under consideration. Structural analysis is a powerful tool for determining diagnosis possibilities and evaluating whether the number and the location of sensors are adequate in order to meet some diagnosis specifications. The proposed approach is successfully applied to leakage detection in a Drinking Water Distribution Network.
\end{abstract}

\section{INTRODUCTION}

Fault diagnosis is of great importance for distribution network systems. It represents an important factor for quality service (related with fast maintenance response to fault situations), in water and electrical distribution networks. In these systems, it is obvious that only a limited number of sensors can be installed due to budget constraints. Since improper selections may seriously hamper diagnosis performance, the development of a sensor placement strategy has become an important research issue in recent years. Ideally, a sensor network should be configured to facilitate fault detection and maximize diagnosis performance under a given sensor cost limit.

Some works devoted to sensor placement for diagnosis using graph tools can be found in [1], [2], [3], [4], [5] and [6] . All these works use a structural model-based approach and define different diagnosis specifications to solve the sensor placement problem. A structural model is a coarse model description, based on a bi-partite graph, that can be obtained early in the development process, without major engineering efforts. This kind of model is suitable to handle large scale systems since efficient graph-based tools can be used and does not have numerical problems. Structural analysis is a powerful tool for early determination of fault diagnosis performances [7].

This work has been funded by the Spanish Ministry of Science and Technology through the CICYT project WATMAN (ref. DPI2009-13744), by the Spanish Ministry of Economy and Competitiveness through the CICYT projecte SHERECS (ref. DPI2011-26243), and by the European Commission through contract i-Sense (ref. FP7-ICT-2009- 6-270428).

F. Nejjari and R. Sarrate are with the Automatic Control Department, Universitat Politècnica de Catalunya, Rambla de Sant Nebridi, 10, 08222 Terrassa, Spain. email: ramon.sarrate, fatiha.nejjari@upc.edu

A.Rosich is with the Institute of Robotics and Industrial Informatics, Edifici U, Pau Gargallo, 5, 08028-Barcelona, Spain. email: arosich@iri.upc.edu
This paper presents a new sensor placement algorithm based on an extension of the work done in [8] that takes into account maximum diagnosability specifications. The sensor placement methodology is applied to a real District Metered Area (DMA) network located in Barcelona. The goal consists in finding the best diagnosis performance that can be achieved by installing a specific number of sensors. The strategy is based on the structural model of the water distribution network. In particular, the present paper proposes the study of which pressure sensors need to be installed in order to improve and maximize the capability of detecting and isolating leaks in the DMA. Water loss in distribution networks is an issue of great concern for water utilities, strongly linked with operational costs and water resource savings.

Usually a leakage detection method in a DMA starts analyzing input flow data, such as minimum night flows and consumer metering data. Once the water distribution district is identified to have a leakage, techniques are used to locate the leakage for pipe replacement or repairing. The whole process could take weeks or months with an important volume of wasted water.

Techniques based on locating leaks from pressure monitoring devices allow a more effective and less costly search in situ. In [9] a leakage localization method based on the pressure measurements and sensitivity analysis of nodes in a network has been proposed. In order to maximize the isolability with a reasonable number of sensors an optimal sensor placement methodology based on genetic algorithms is also proposed. The optimization goal consisted in minimizing the size of the larger set of non-isolable leaks.

The paper is organized as follows: In Section II, the sensor placement problem tackled in this paper is presented. Section III formally introduces the diagnosis framework based on structural models. Section IV describes the algorithm used to solve the aforementioned problem. In Section V, the sensor placement methodology is applied to a real DMA network. Finally, some conclusions and remarks are given in Section VI.

\section{Problem formulation}

Usually, the sensor placement problem is presented as an optimization problem where the best sensor configuration fulfilling some given diagnosis specifications is sought, see e.g. [10] and [11]. Nevertheless, in our case, the water supply company is not interested in the best sensor configuration but in the best diagnosis performance that can be achieved by installing a specific number of sensors. Therefore, this paper 
introduces a slightly different problem formulation motivated by practical reasons.

Let $\mathbf{S}$ be the candidate sensor set and $m$ the number of sensors that will be installed in the system. Then, the problem can be roughly stated as the choice of a combination of $m$ sensors in $\mathbf{S}$ such that the diagnosis performance is maximised.

In model-based diagnosis, fault detectability and fault isolability are the main objectives. Fault detectability is the ability of monitoring a fault occurrence in a system, whereas fault isolability concerns the capacity of distinguishing between two possible fault occurrences. Thus, the diagnosis performance will be stated based on fault detectability and isolability properties. In this work, the single fault assumption will hold (i.e., multiple faults will not be covered) and no candidate sensor fault will be considered. Moreover, in this case, faults will represent leaks in the water distribution network.

Let $\mathbf{F}$ be the set of faults that must be monitored, then $F_{D}(S) \subseteq \mathbf{F}$ denotes the detectable fault set assuming that a sensor configuration $S \subseteq \mathbf{S}$ is installed in the system. Fault isolability can be characterised in a similar way by means of fault pairs. For the sake of simplicity, it is assumed that all isolable faults are detectable; this implies that the symmetry property [2] holds for any pair of isolable faults (i.e., if $f_{i}$ is isolable from $f_{j}$ then $f_{j}$ is isolable from $f_{i}$ ). Let $\mathbb{F}: \mathbf{F} \times \mathbf{F}$ be all fault pairs from $\mathbf{F}$, then $\mathcal{F}_{I}(S) \subseteq \mathbb{F}$ denotes the set of isolable fault pairs when the sensor configuration $S \subseteq \mathbf{S}$ is chosen for installation (i.e., $\left(f_{i}, f_{j}\right) \in \mathcal{F}_{I}(S)$ means that fault $f_{i}$ is isolable from $f_{j}$ when the sensor set $S$ is installed in the system). Note that due to the symmetry property, only combinations of two faults need to be considered, instead of permutations of two faults.

Based on $\mathcal{F}_{I}(S)$, the isolability index $I(S)$ is defined as the number of isolable fault pairs when the sensor configuration $S$ is installed, i.e.,

$$
I(S)=\left|\mathcal{F}_{I}(S)\right|
$$

where $|\cdot|$ denotes the cardinality of the set.

To solve the sensor placement problem proposed in this paper, a system description $\mathcal{M}$ is also required. Such description will allow the computation of the detectable faults and the isolability index for a given sensor configuration. Hence, the sensor placement for fault diagnosis can be formally stated as follows:

GIVEN a candidate sensor set $\mathbf{S}$, a system description $\mathcal{M}$, a fault set $\mathbf{F}$, and the number $m$ of sensors to be installed.

FIND the $m$-sensor configuration $S \subset \mathbf{S}$ such that:

1) all faults in $\mathbf{F}$ are detectable, $F_{D}(S)=\mathbf{F}$, and

2) the number of isolable fault pairs is maximised, i.e. $I(S) \geq I\left(S^{\prime}\right)$ for any $S^{\prime} \subseteq \mathbf{S}$ such that $\left|S^{\prime}\right|=m$.

By solving this problem for the water distribution network, all leaks are guaranteed to be detectable, and no other sensor configuration involving $m$ sensors has better isolability capabilities. These properties make the solution of this problem very attractive from the water supply company perspective. It is worth noting that other diagnosis performance indexes, also designed for sensor placement, could be used here, see for example [9] and [5]. However, these indexes may fail at representing maximum fault isolability.

The objective of this paper is to derive an algorithm that computes a solution for the aforementioned problem. This algorithm will perform a search over different sensor configurations until a solution is found.

\section{FAULT DIAGNOSIS BASED ON STRUCTURAL MODELS}

A structural model approach will be used to solve the sensor placement problem stated in the previous section. The analysis of the model structure has been widely used in the area of model-based fault diagnosis [7]. Therefore, consistent tools exist in order to perform diagnosability analysis and consequently compute the set of detectable and isolable faults.

The structural model is often defined as a bipartite graph $G(M, X, A)$, where $M$ is a set of model equations, $X$ a set of unknown variables and $A$ a set of edges, such that $\left(e_{i}, x_{j}\right) \in A$ as long as equation $e_{i} \in M$ depends on variable $x_{j} \in X$. A structural model is a graph representation of the analytical model structure since only the relation between variables and equations is taken into account, neglecting the mathematical expression of this relation.

Structural modelling is suitable for an early stage of the system design, when the precise model parameters are not known yet, but it is possible to determine which variables are related to each equation. Furthermore, the diagnosis analysis based on structural models is performed by means of graphbased methods which have no numerical problems and are more efficient, in general, than analytical methods. However, due to its simple description, it cannot be ensured that the diagnosis performance obtained from structural models will hold for the real system. Thus, only best case results can be computed.

It is well-known that the over-determined part of the model is the only useful part for system monitoring [7]. The Dulmage-Mendelsohn (DM) decomposition [12] is a bipartite graph decomposition that defines a partition on the set of model equations $M$. It turns out that one of these parts is the over-determined part of the model and is represented as $M^{+}$.

The system fault diagnosis analysis is next performed based on the structural model properties. Specifically, fault detectability and isolability are defined as properties of the over-determined part of the model [2]. First, it is assumed that a single fault $f \in \mathbf{F}$ can only violate one equation (known as fault equation), denoted by $e_{f} \in M$.

Definition 1: A fault $f \in F$ is (structurally) detectable in a model described by the set of equations $M$ if

$$
e_{f} \in M^{+}
$$


Definition 2: A fault $f_{i}$ is (structurally) isolable from $f_{j}$ in a model described by the set of equations $M$ if

$$
e_{f_{i}} \in\left(M \backslash\left\{e_{f_{j}}\right\}\right)^{+}
$$

Without loss of generality, it is assumed that a sensor $s_{i} \in \mathbf{S}$ can only measure one single unknown variable $x_{i} \in X$. In the structural framework, such sensor will be represented by one single equation denoted as $e_{s}$ (known as sensor equation). Given a set of sensors $S$, the set of sensor equations is denoted as $M_{S}$. Thus, given a candidate sensor configuration $S$ and a model $M$, the updated system model corresponds to $M \cup M_{S}$.

From Definition $1, F_{D}(S)$ can be computed as

$$
F_{D}(S)=\left\{f \in \mathbf{F} \mid e_{f} \in\left(M_{S} \cup M\right)^{+}\right\}
$$

and from Definition 2, $\mathcal{F}_{I}(S)$ can be computed as

$$
\mathcal{F}_{I}(S)=\left\{\left(f_{i}, f_{j}\right) \in \mathbb{F} \mid e_{f_{i}} \in\left(M_{S} \cup\left(M \backslash\left\{e_{f_{j}}\right\}\right)\right)^{+}\right\}
$$

It is worth noting that testing different sensor configurations involves different sensor equation sets, $M_{S}$, in (4) and (5).

Remark that the isolability index, $I(S)$ can be computed straight away as the number of elements in $\mathcal{F}_{I}(S)$, according to (1).

\section{OPTIMAL SENSOR PLACEMENT ALGORITHM}

The sensor placement problem stated in Section II involves finding an $m$-sensor configuration among $|\mathbf{S}|$ candidate sensors. One trivial approach to solve this problem would be to check all these $\left(\begin{array}{c}|\mathbf{S}| \\ m\end{array}\right)$ sensor configurations. However, such search would be highly inefficient.

Alternatively, the optimal sensor placement problem will be solved by Algorithm 1, which is based on a depth-first branch and bound search. This search is expected to perform better than the trivial approach. Later, in Section V, some performance issues will be discussed.

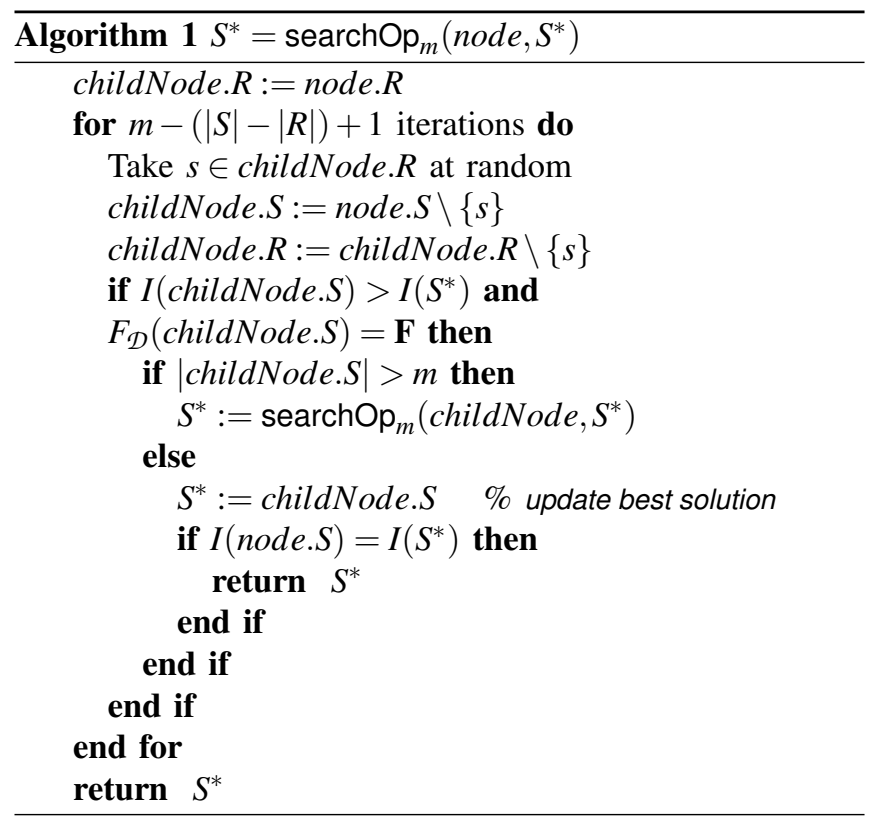

Every node in the search tree consists of two sensor sets:
- node.S, the sensor configuration that the node represents.

- $n o d e . R$, the set of sensors that are allowed to be removed in its child nodes.

Throughout the search, the best solution is updated in $S^{*}$ whenever a feasible $m$-sensor configuration with a higher fault isolability index than the current best one is found. A branch operation is initiated whenever a feasible sensor configuration with a higher fault isolability index is found, as long as the sensor configuration involves more than $m$ sensors. Initiating a branch operation involves a recursive call to searchOp ${ }_{m}$.

A branch operation is aborted at some child node whenever any of the following three conditions holds:

C1A: The fault isolability index corresponding to the node does not improve the current best one.

C2A: The node does not correspond to a feasible sensor configuration. A feasible configuration means a sensor configuration such that all $f \in \mathbf{F}$ are detectable.

C3A: The node corresponds to a feasible $m$-sensor configuration that improves the current best fault isolability index.

A branch operation always involves removing a sensor from a sensor configuration, so if condition $\mathrm{C} 1 \mathrm{~A}$ holds then no sub-node can improve the best isolability index either. Moreover, if condition $\mathrm{C} 2 \mathrm{~A}$ holds then no sub-node corresponds to a feasible sensor configuration either. Lastly, condition $\mathrm{C} 3 \mathrm{~A}$ implies that this node corresponds to a local solution. Hence, no sub-node will correspond to a local solution, since it would involve less that $m$ sensors.

A branch operation involves visiting the child nodes of a parent node. Aborting a branch operation at a parent node means that a call to searchOp ${ }_{m}$ returns. A branch operation is aborted at a parent node when any of the following two conditions hold:

C1B: All child nodes that are ancestors of some $m$-sensor configurations have been already visited.

C2B: A local solution has been found with the same fault isolability index than that of the parent node.

Condition $\mathrm{C} 1 \mathrm{~B}$ holds when the first $m-(|S|-|R|)+1$ child nodes have been visited. So, visiting the rest of the child nodes is not worth it. On the other hand, if condition C2B holds, no other child node is expected to improve the current best isolability index.

Remark that in a branch operation child nodes are visited at random order. Apparently, following a decreasing child node isolability index order would be more efficient. However, this strategy would entail the need of computing the isolability index of all the child nodes of a given node at every branch operation, which would result in an efficiency penalty.

Algorithm 1 is initialised as follows:

1) The root node of the search tree corresponds to the candidate sensor set: node. $S:=$ node. $R .=\mathbf{S}$. 
2) The current best sensor configuration corresponds to the empty set: $S^{*}:=\emptyset$.

\section{APPLICATION TO A WATER DISTRIBUTION NETWORK}

This section shows how to solve the aforementioned problem for the case of a specific water distribution network. First, the DMA network is introduced where the most relevant features from the diagnosis perspective are given. Then, the structural model representation of this network is presented. And, finally the sensor placement problem is solved, obtaining encouraging results.

A leakage detection method involves dividing the distribution system into well-defined DMAs. Leakage level in a DMA is determined based on the minimum night flow minus the legitimate night demand and estimated unavoidable background leakage. DMAs help identifying areas of the pipe network that suffer from excessive leakage.

\section{A. Water network description}

The sensor placement methodology is applied to a DMA located in Barcelona area (see Figure 1). It has 881 nodes and 927 pipes. The network consists of 311 nodes with demand (RM type), 60 terminal nodes with no demand (EC type), 48 nodes hydrants without demand (HI type), 14 dummy valve nodes without demand (VT type) and 448 dummy nodes without demand (XX type). The set of dummy nodes represents the defects causing leaks in the network. The network has two inflow inputs modeled as reservoir nodes.

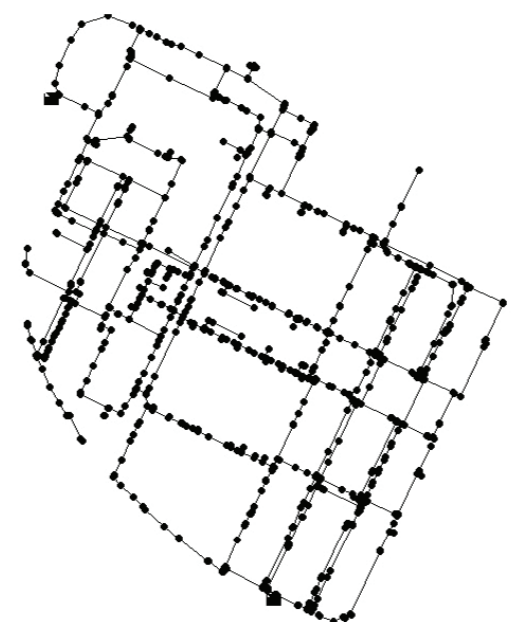

Fig. 1. Case study network map

Leakage detection is based on the premise that damage (leakage) in one or more locations of the piping network involves local liquid outflow at the leakage location, which will change the flow characteristics (pressure heads, flow rates, acoustics signals, etc.) at the monitoring locations of the piping network.

Leaks might appear anywhere in the water network. However, due to simulation limitations, leaks are represented in the nodes where the flow balances take place. Therefore, a node with a leak can be modeled for simulation as

$$
\sum q_{\text {in }}-\sum q_{\text {out }}=q_{f}
$$

where $q_{\text {in }}$ are the input flows, $q_{\text {out }}$ are the output flows and $q_{f}$ is the outflow caused by the leak. In this case $q_{f}$ is considered as an unknown input of the system.

Only dummy nodes can have leaks. Thus, since there are 448 dummy nodes (XX type) in the network, there are 448 potential leaks to be detected and isolated.

Hydraulic sensors may monitor pressure or flow rate. This work focuses on the placement of pressure monitoring points as they are more frequently used than flow rate sensors. Collecting pressure data is cheaper and easier, and the pressure transducers give instantaneous readings whereas most flow meters do not react instantaneously to flow changes [13]. Flow rates are usually measured at all entry points to the network; on main pipes at the entrance into subnetworks; and/or at the outlet of elevated tanks and pumping stations. Thus, the selection of flow rate measurement points is straightforward and is limited to specific locations.

Therefore, only pressure sensors will be considered in the sensor placement problem. In order to reduce the problem complexity, just a subset of pressures is chosen as candidate variables to be measured. Identifying the optimal locations of these sensors is important from an economic perspective.

\section{B. Structural model extraction}

As it was shown in Section III, the fault diagnosis framework is based on structural models. Next, it will be explained how the corresponding structural model of the DMA water network is systematically obtained from the network graph.

The DMA network is originally represented as a directed graph $G=(N, E)$ where pipe junctions are nodes, $N$, and pipes are edges, $E$. Each node represents, at the same time, a pressure variable and a flow balance equation. Similarly, each edge represents a flow variable and a pipe equation. Therefore, given a node $n \in N$, the following flow balance equation can be derived,

$$
\sum_{q_{i} \in Q_{n}} q_{i}=d_{n}
$$

where $Q_{n}$ represents all the flows of the edges incident to node $n$, and $d_{n}$ is the known flow demand associated to node $n$. Furthermore, given an edge $e \in E$, the corresponding pipe equation can be deduced as

$$
q_{e}=\operatorname{sgn}\left(p_{i}-p_{j}\right) \cdot c\left(\left|p_{i}-p_{j}\right|\right)^{\gamma}
$$

where $q_{e}$ is the flow of edge $e, p_{i}$ and $p_{j}$ are the pressures of the nodes adjacent to edge $e=\left(n_{i}, n_{j}\right)$, and $c$ and $\gamma$ are parameters modelling physical properties of the pipe, such as length, inside diameter, minor losses, and roughness.

Now, the structural model of the water network can be defined as a bipartite graph involving the equation node set $M$ and the unknown variable node set $X$. Lets $M_{N}$ be the set of flow balance equations and $M_{E}$ be the set of pipe equations, then $M=M_{N} \cup M_{E}$. Note that there are as many equations in $M_{N}$ as nodes in $G$ and as many equations in $M_{N}$ as edges in $G$. This amounts to 1810 equations for the Barcelona DMA network used here. On the other hand, let $Q$ be the set of flow variables and $P$ the set of pressure variables, then it 


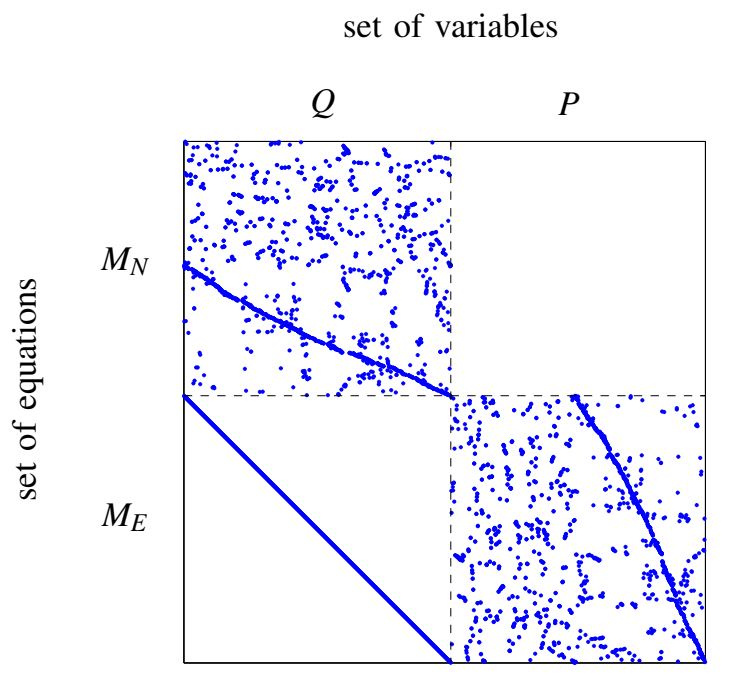

Fig. 2. Structural model of the DMA network

holds that $X=Q \cup P$. The number of unknown variables is therefore 1810. The edges of the structural model are defined from the graph $G$, according to (7) and (8). In Figure 2, the resulting structural model is depicted in matrix form where the equation set corresponds to rows and the variable set corresponds to columns. A dot in the $(i, j)$ element indicates that there exists an edge incident to equation $e_{i} \in M$ and variable $x_{j} \in X$, i.e., $\left(e_{i}, x_{j}\right) \in A$. Note that the structural model of the DMA network is a just-determined model where all unknown variables can be computed, i.e. the model can be used for system simulation.

\section{Sensor placement for leakage detection and isolation}

It is important to see that when a leak is present in a dummy node (type XX), then the corresponding equation of the form in (7) does no longer hold. Indeed, a term $q_{f}$ should be added to the equation so that the model becomes consistent with the faulty water network. However, since detecting inconsistencies in the equation is the objective of model-based diagnosis, the term $q_{f}$ is omitted and the set of faults, or leaks, is now represented as the subset of structural model equations in $M_{N}$ related to dummy nodes. Therefore, the following set of fault equations is defined

$$
M_{F}=\left\{e \in M_{N} \mid e \text { comes from a node } \mathrm{XX}\right\}
$$

Up to 31 pressure variables from $P$ have been chosen as the candidate sensor set $\mathbf{S}$. When a sensor measuring pressure $p_{i}$ is placed, the equation $p_{i}=\hat{p}_{i}$ is added to the structural model, where $\hat{p}_{i}$ is the measurement obtained from the sensor reading.

If all candidate sensors were installed, the maximum diagnosis performance would be achieved. Recall that, in this application, the fault set $\mathbf{F}$ has a cardinality of 448 elements (i.e., the number of type XX nodes). So, the maximum isolability index would ideally be $\left(\begin{array}{c}448 \\ 2\end{array}\right)=100128$. However, when installing all candidate sensors, all leaks
TABLE I

OPTIMAL SENSOR PLACEMENT SOLUTION

\begin{tabular}{|c|c||c|c|}
\hline Sensor & Sensor ID & Sensor & Sensor ID \\
\hline S1 & RM00091308 & S5 & RM00097476 \\
S2 & RM00091309 & S6 & RM00097508 \\
S3 & RM00091329 & S7 & RM00211544 \\
S4 & RM00097475 & S8 & RM00215963 \\
\hline
\end{tabular}

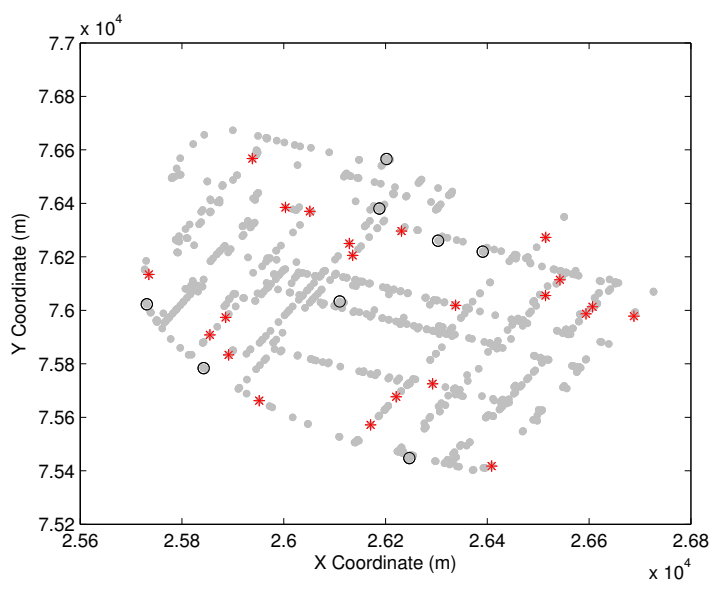

Fig. 3. DMA network with the optimal sensor location (black $\circ$ symbol) and the remaining candidate sensors (red $*$ symbol)

can be detected but the isolability index is just 100099 . Achieving the ideal isolability index would require installing more sensors than those designated in the candidate sensor set. Therefore, there is a trade-off between the diagnosis performance and the number of sensors required, which in the end results in a cost penalty.

Assume that the water distribution company has established a maximum budget for investment on instrumentation that makes it possible to install up to 8 sensors. Hence, the water distribution company wants to install 8 sensors such that the maximum diagnosis performance can be achieved. Algorithm 1 is applied to solve this problem with $m:=$ 8. After 256 seconds, the algorithm returns the 8-sensor configuration shown in Table I. With these 8 sensors all leaks can be detected and the isolability index amounts to 100092 .

The optimal sensor configuration is depicted in Figure 3 where nodes corresponding to the optimal measurements as well as those corresponding to the candidate sensor set are highlighted.

In order to illustrate the trade-off between the best achievable isolability index and $m$, Algorithm 1 has been run with different values for $m$. Figure 4 shows these results. Remark that all optimal sensor configurations with more than 12 sensors achieve the same maximum isolability index than the 31 candidate sensor configuration. Any sensor configuration with less than 13 sensors involves decreasing the isolability index. On the other hand, a sensor configuration with less than 2 sensors does not satisfy the full fault detectability specification, so it is no included in the figure.

Regarding the search strategy performance issues when $m:=8$, the trivial approach introduced at the beginning of 


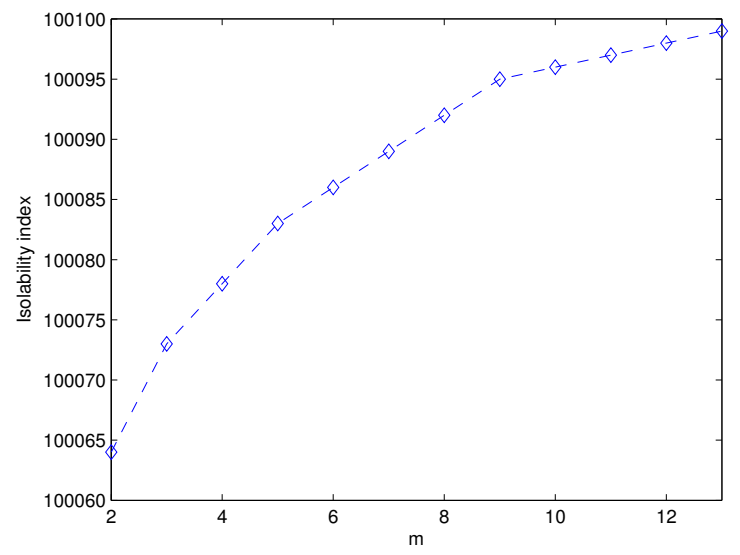

Fig. 4. Fault diagnosis performance tradeoff

Section IV would involve computing the isolability index of about 8 million sensor configurations (i.e., $\left(\begin{array}{c}31 \\ 8\end{array}\right)$ ). However, applying Algorithm 1 the isolability index is just computed 17286 times, which definitively saves a lot of time.

\section{Conclusions}

The sensor placement problem in water distribution networks has been addressed in this paper. However, the method could also be applied to other distribution networks such as those related to gas, electricity, etc. A distribution network usually describes a mesh topology involving hundreds of interconnected nodes whose behaviour follows non-linear physical laws. Such complexity requires the development of tools applicable to non-linear large-scale systems. This paper applied structural analysis framework to address this complexity.

A key contribution of this work is the definition of the isolability index as a measurement of the fault diagnosis performance achievable in a given system. This measurement allows to set up a sensor placement problem based on a fault diagnosis performance maximization criterium. This is an original contribution since, in the literature, most approaches to optimal sensor placement try to solve a different problem: search the minimum cost sensor configuration that satisfies a given set of fault diagnosis specifications.

The new formulation presented in this paper becomes appropriate in distribution networks, where the budget assigned to instrumentation is limited and all sensors usually measure the same kind of variable. A possible extension would involve considering various types of sensors with different costs. This would lead to a more challenging problem: fault diagnosis performance maximization under the constraint of a given maximum sensor configuration cost.

In model-based fault diagnosis, diagnosis is basically performed based on the response of residual generators, which are derived from the model equations. When the model includes nonlinearities, deriving a residual generator can become a difficult or even a practically infeasible task. In this paper, this issue has been ignored. However, functions $F_{D}(S)$ and $I(S)$ could be adapted to take into account this constraint in the sensor placement analysis phase, by following the causality framework introduced in [14]. Then, the solution obtained from the sensor placement analysis would guarantee a set of easily computable residual generators.

\section{REFERENCES}

[1] R. Raghuraj, M. Bhushan, and R. Rengaswamy, "Locating sensors in complex chemical plants based on fault diagnostic observability criteria," AIChE J., vol. 45, no. 2, pp. 310-322, Feb. 1999.

[2] M. Krysander and E. Frisk, "Sensor placement for fault diagnosis," IEEE Trans. Syst., Man, Cybern. A, vol. 38, no. 6, pp. 1398-1410, 2008.

[3] C. Commault, J. M. Dion, and S. Y. Agha, "Structural analysis for the sensor location problem in fault detection and isolation," Automatica, vol. 44, no. 8, pp. 2074-2080, aug 2008.

[4] A. A. Yassine, S. Ploix, and J. M. Flaus, "A method for sensor placement taking into account diagnosability criteria," Int. J. Appl. Math. Comput. Sci., vol. 18, no. 4, pp. 497-512, 2008.

[5] L. Travé-Massuyès, T. Escobet, and X. Olive, "Diagnosability analysis based on component supported analytical redundancy relations," IEEE Trans. Syst., Man, Cybern. A, vol. 36, no. 6, pp. 1146-1160, 2006.

[6] A. Rosich, R. Sarrate, V. Puig, and T. Escobet, "Efficient optimal sensor placement for model-based FDI using and incremental algorithm," in Proc. 46th IEEE Conference on Decision and Control, New Orleans, USA, Dec. 12-14, 2007, pp. 2590-2595.

[7] M. Blanke, M. Kinnaert, J. Lunze, and M. Staroswiecki, Diagnosis and Fault-Tolerant Control, 2nd ed. Springer, 2006.

[8] A. Rosich, A. A. Yassine, and S. Ploix, "Efficient optimal sensor placement for structural model based diagnosis," in 21th International Workshop on Principles of Diagnosis (DX-10), Portland, USA, October 2010.

[9] R. Pérez, V. Puig, J. Pascual, J. Quevedo, E. Landeros, and A. Peralta, "Methodology for leakage isolation using pressure sensitivity analysis in water distribution networks," Control Engineering Practice, vol. 19, no. 10 , pp. 1157 - 1167, 2011.

[10] M. Bagajewicz, A. Fuxman, and A. Uribe, "Instrumentation network design and upgrade for process monitoring and fault detection," AIChE J., vol. 50, no. 8, pp. 1870-1880, Aug. 2004.

[11] A. Rosich, R. Sarrate, and F. Nejjari, "Optimal sensor placement for fdi using binary integer linear programming," in 20th International Workshop on Principles of Diagnosis (DX-09), Stockholm, Sweden, June 2009.

[12] A. L. Dulmage and N. S. Mendelsohn, "Covering of bi-partite graph," Canada J. Math, vol. 10, pp. 527-534, 1958.

[13] W. de Schaetzen, G. A. Walters, and D. A. Savic, "Optimal sampling design for model calibration using shortest path, genetic,and entropy algorithms," Urban Water, vol. 2, pp. 141-152, 2000.

[14] A. Rosich, E. Frisk, J. Åslund, R. Sarrate, and F. Nejjari, "Fault diagnosis based on causal computations," IEEE Trans. Syst., Man, Cybern. A, vol. 42, no. 2, pp. 371-381, March 2012. 